\title{
Avaliação de interações medicamentosas potenciais em prescrições da atenção primária de Vitória da Conquista (BA), Brasil
}

\author{
Evaluation of potential drug interactions in primary health care \\ prescriptions in Vitória da Conquista, Bahia (Brazil)
}

Danyllo Fábio Lessa Leão ${ }^{1}$

Cristiano Soares de Moura ${ }^{1}$

Danielle Souto de Medeiros ${ }^{1}$

${ }^{1}$ Instituto Multidisciplinar em Saúde, Universidade Federal da Bahia. R. Rio de Contas 58/Quadra 17/Lote 58/Pavilhão de Aulas IMS/ UFBA, Candeias. 45.029-094 Vitória da Conquista BA Brasil. danyllolessa@gmail.com
Abstract Drug interactions are risk factors for the occurrence of adverse drug reactions. The risk for drug interactions includes factors related to prescription that are intrinsic to the patient. This study sought to evaluate the potential drug interactions in primary care prescriptions in Vitória da Conquista in the state of Bahia to fill the knowledge gap on this topic in Brazil. Information about several variables derived from the primary health care prescriptions was collected and drug interactions were evaluated based on information from Medscape and Micromedex ${ }^{\circledR}$ databases. Polypharmacy frequency and its association with the occurrence of drug interactions were also evaluated. Results revealed a 48,9\% frequency of drug interactions, 74,9\% of moderate or greater severity, 8,6\% of prescriptions in polypharmacy that in the chi-square test showed a positive association with the occurrence of drug interactions ( $p$ $<0,001)$. Prescriptions from primary care in Vitória da Conquista in the state of Bahia showed a high frequency of drug interactions, however it is necessary to analyze other risk factors for their occurrence at this level of health care.

Key words Drug interactions, Polypharmacy, Primary health care, Prescription quality
Resumo As interações medicamentosas são fatores de risco para a ocorrência de reações adversas a medicamentos. Este estudo teve o objetivo de avaliar as interações medicamentosas potenciais em prescrições da atenção primária de Vitória da Conquista (BA), visando preencher a lacuna de conhecimento sobre essa temática no Brasil. Foram coletadas informações sobre diversas variáveis de prescrições oriundas da atenção primária $e$ as interações medicamentosas avaliadas a partir dos bancos de dados do Medscape e Micromedex ${ }^{\circledR}$. Verificou-se ainda a frequência de polifarmácia e associação desta com a ocorrência de interações medicamentosas. Os resultados mostraram frequência de 48,9\% de interações medicamentosas, 74,9\% delas de gravidade moderada ou maior, e 8,6\% de prescrições em polifarmácia que, em teste qui-quadrado, mostrou associação positiva com ocorrência de interações medicamentosas potenciais $(p<0,001)$. As prescrições oriundas da atenção primária de Vitória da Conquista (BA) apresentaram uma alta frequência de interações medicamentosas, porém faz-se necessária a análise de outros fatores de risco para ocorrência destas nesse nível de atenção à saúde.

Palavras-chave Interação medicamentosa, Polifarmácia, Atenção Primária, Qualidade de prescrição 


\section{Introdução}

O conceito de interação medicamentosa baseiase na resposta farmacológica ou clínica oriunda da interferência da ação de um determinado medicamento, alimento ou qualquer substância química sobre o efeito de outro medicamento, administrado previamente ou em concomitância ao primeiro ${ }^{1,2}$.

As interações entre medicamentos podem ser classificadas em sinérgicas, quando o efeito da interação é maior que o efeito individual dos medicamentos, e antagônicas, quando o efeito da interação é menor que o efeito individual dos medicamentos ou quando há alteração/anulação da resposta farmacológica dos mesmos. O mecanismo de interação medicamentosa pode ser de caráter físico-químico, farmacodinâmico ou farmacocinético ${ }^{3}$.

Diversos são os fatores de risco para a ocorrência de interações medicamentosas, sendo estes relacionados à prescrição, onde o aumento do risco de interações é diretamente proporcional à quantidade de medicamentos prescritos condições intrínsecas ao paciente, como idade, sexo e condições de saúde; e fatores intrínsecos ao medicamento, principalmente o índice terapêutico. A presença de um ou mais fatores de risco de interação medicamentosa aumenta a complexidade de uma prescriçãa $0^{5,6}$.

Interações medicamentosas potenciais, capazes de resultar em reações adversas, constituem um importante indicador de qualidade de prescrição. Esse indicador é mensurado pelo grau de polifarmácia do indivíduo, ou seja, pela quantidade de medicamentos contidos na prescrição ${ }^{7}$.

Em 2010, Fröhlich et al. evidenciaram que o aumento da complexidade da prescrição suscetibiliza o indivíduo a interações medicamentosas, possíveis reações adversas, surgimento de comorbidades e, consequentemente, diminuição da sua qualidade de vida ${ }^{6}$.

Dados encontrados na literatura revelaram que a incidência de internações hospitalares em decorrência de interações medicamentosas pode chegar a $2,8 \%^{8}$.

A maior parte das investigações sobre interações medicamentosas foi conduzida em ambiente hospitalar ${ }^{2,9,10}$. Por outro lado, no Brasil, há lacunas de conhecimento sobre a ocorrência de interações na atenção primária à saúde. De fato, devido às particularidades desse nível de atenção, onde é habitual que os pacientes manifestem sinais e sintomas precoces das enfermidades, que muitas vezes implicam em condutas e tratamentos empíricos ${ }^{11}$, torna-se relevante a realização de estudos acerca desse tema.

O presente trabalho objetivou avaliar interações medicamentosas potenciais através da análise de prescrições da atenção primária de Vitória da Conquista (BA).

\section{Métodos}

Este trabalho faz parte da pesquisa intitulada "Avaliação de prescrições dispensadas em Unidades de Saúde em Vitória da Conquista (BA)", inserida no Programa Educação pelo Trabalho (PET) - Saúde da Família do Ministério da Saúde. Trata-se de um estudo de delineamento transversal, realizado na farmácia da Unidade de Saúde da Família Urbis V, localizada na zona urbana de Vitória da Conquista (BA). Os dados foram obtidos dos registros de prescrições atendidas na referida unidade no período de julho a dezembro de 2010.

Somente foram incluídas as prescrições com segundas vias arquivadas e separadas por mês e ano em que foram atendidas. Prescrições que continham um único medicamento foram excluídas, por não apresentarem risco de interação medicamentosa. A partir do universo de 2399 prescrições do período, foi selecionada uma amostra probabilística, cujo tamanho foi calculado com base nos seguintes parâmetros: frequência antecipada do evento de $50 \%$, precisão estimada em 5\%, intervalo de confiança de $95 \%$ e efeito de desenho igual a 1 , resultando em uma amostra de 332 prescrições. A esse número foi acrescentado $10 \%$ para antecipar eventuais perdas, o que resultou em 365 prescrições.

As informações coletadas nas prescrições foram: nome do medicamento, identificação da via de administração, identificação da forma farmacêutica, dose, frequência, duração do tratamento e recomendações de uso. Foram coletados dados de todos os medicamentos prescritos, mas a análise de interações excluiu os fitoterápicos e os princípios ativos como vitaminas, sais minerais e componentes da dieta.

Para avaliação de interações medicamentosas potenciais, foi utilizada parte da metodologia descrita no trabalho de Moura et al. ${ }^{2}$. As interações detectadas neste estudo são consideradas potenciais porque expressam a possibilidade de ocorrência de uma interação, cuja manifestação clínica pode ter ocorrido ou não ${ }^{12}$. As interações medicamentosas foram identificadas a partir de duas bases de dados informatizadas, o Drug In- 
teraction Checker ${ }^{13}$, do Medscape e o University of Maryland Medical Center Drug Checker ${ }^{14}$, base de dados do Micromedex ${ }^{\circledR}$. Foram consideradas as interações fármaco-fármaco contidas em uma mesma prescrição. Nos casos de discordância entre as bases quanto à classificação de interação, foi adotada a de menor gravidade.

A partir das informações disponíveis nas bases consultadas, as interações medicamentosas foram descritas quanto às reações adversas potenciais e classificadas quanto à gravidade (menor ou não significante, moderada ou significante e maior ou muito significante), que leva em consideração o risco de efeitos clínicos, e quanto ao mecanismo de interação (em farmacocinético, farmacodinâmico, físico-químico ou misto/ desconhecido).

As prescrições foram classificadas como em polifarmácia com base no número de medicamentos prescritos; foram consideradas em polifarmácia as prescrições que continham cinco ou mais medicamentos ${ }^{15}$.

Os dados foram armazenados em software QDS (Questionnaire Development System), versão 2.6.1. Foram realizadas análises descritivas dos dados através de distribuição de frequência, medidas de tendência central (média \pm desvio padrão ou mediana) e dispersão das diferentes variáveis do estudo. O intervalo de confiança a $95 \%\left(\mathrm{IC}_{95 \%}\right)$ para a frequência de interações foi estimado com base em um método exato (mid$\mathrm{P}$ exato) e o teste qui-quadrado foi empregado para avaliar a associação entre polifarmácia e interação medicamentosa. As análises foram executadas no programa Epi-Info, versão 3.5.3.

$\mathrm{O}$ projeto de pesquisa foi aprovado pelo $\mathrm{Co}-$ mitê de Ética em Pesquisas da Secretaria Estadual de Saúde do Estado da Bahia, de acordo com a resolução No 196/96 do Conselho Nacional de Saúde ${ }^{16}$. Termo de consentimento da instituição foi obtido para permitir o acesso às prescrições dos usuários. Foi garantida a confidencialidade das informações, não havendo risco de divulgação dos dados pessoais obtidos a partir das prescrições.

\section{Resultados e discussão}

Foram analisadas 350 prescrições, que apresentaram média de 2,8 $\pm 1,2$ medicamentos por prescrição. Os medicamentos mais comumente prescritos estão dispostos na Tabela $1 \mathrm{e}$ incluem ibuprofeno $(13,3 \%)$, paracetamol $(9,5 \%)$, amoxicilina $(7,0 \%)$, dexclorfeniramina $(4,8 \%)$ e hidro- clorotiazida (3,6\%). Ibuprofeno, paracetamol, omeprazol e hidroclorotiazida também estavam entre os dez medicamentos mais prescritos em um estudo realizado na atenção primária da Região da Múrcia (Espanha). Os autores ainda relatam que estes, exceto o paracetamol, estão entre os seis medicamentos mais frequentemente implicados em interações graves, por isso foram incluídos em uma escala com classificação de perigo alto e extremo ${ }^{17}$.

Dentre os medicamentos analisados $(\mathrm{N}=$ 975), 35,0\% apresentaram a via de administração registrada na prescrição, $75,9 \%$ a forma farmacêutica, $75,2 \%$ tinham dose do medicamento especificada, 96,4\% apresentaram frequência de uso, $39,6 \%$ indicavam duração do tratamento e 53,2\% recomendações de uso para o medicamento.

É preocupante a baixa frequência de informações sobre a duração de tratamento e recomendações de uso nas prescrições analisadas, uma vez que a ausência desses itens pode resultar em tempo e forma de uso inadequado, aumentando os riscos de ocorrência de efeitos não desejados, como a intoxicação medicamentosa, interações e reações adversas a medicamentos. Observou-se ainda que para a maior parte dos medicamentos prescritos a via de administração não havia sido especificada e para aproximadamente $1 / 4$ dos itens a forma farmacêutica estava ausente. Tais falhas podem predispor a erros de dispensação, que podem interferir diretamente na segurança e eficácia do tratamento farmacológico ${ }^{18,19}$. Resultados semelhantes foram encon-

Tabela 1. Medicamentos mais frequentes em prescrições da Unidade de Saúde da Família Urbis V, Vitória da Conquista, Bahia, Brasil, julho a dezembro de 2010 .

\begin{tabular}{lrr}
\hline \multicolumn{1}{c}{ Fármaco } & N & \multicolumn{1}{c}{$\%$} \\
\hline Ibuprofeno & 130 & 13,3 \\
Paracetamol & 93 & 9,5 \\
Amoxicilina & 68 & 7,0 \\
Dexclorfeniramina & 47 & 4,8 \\
Hidroclorotiazida & 35 & 3,6 \\
Metronidazol & 32 & 3,3 \\
Loratadina & 32 & 3,3 \\
Dipirona & 31 & 3,2 \\
Omeprazol & 29 & 3,0 \\
Enalapril & 20 & 2,1 \\
Cefalexina & 20 & 2,1 \\
Outros & 438 & 44,9 \\
Total & $\mathbf{9 7 5}$ & $\mathbf{1 0 0 , 0}$
\end{tabular}


trados em medicamentos prescritos na atenção primária da Província Riyadh (Arábia Saudita), onde a forma farmacêutica, a dose e a via de administração em $21,7 \%, 8,8 \%$ e $99,6 \%$, respectivamente, não estavam presentes ${ }^{20}$.

A frequência de interações medicamentosas nas prescrições do estudo foi de $48,9 \%$, com média de 0,80 interações por prescrição. Em relação à gravidade, $71,7 \%$ das interações medicamentosas foram consideradas de gravidade moderada (ou significante), sendo suas consequências exigentes de tratamento e monitoramento. Para 3,2\% das interações, a gravidade foi considerada maior (ou de relevância muito significante), situações nas quais pode haver uma reação adversa grave, com risco de morte (Tabela 2). Os pares de medicamentos mais envolvidos em interações incluem os anti-inflamatórios não esteroidais, anti-hipertensivos (inibidores da ECA, diuréticos, $\beta$-bloqueadores), antibióticos e antiasmáticos (Tabela 3).

No âmbito nacional, poucos estudos retrataram o perfil das interações medicamentosas na atenção primária. Em farmácias públicas de São Paulo foram encontradas interações medicamentosas em $8,0 \%$ das prescrições que continham antibióticos. Dessas interações, $57,1 \%$ foram classificadas como interação de baixa gravidade e cerca de $42,9 \%$ consideradas de gravidade mo$\operatorname{derada}^{21}$. A presença de interações medicamentosas também foi descrita em 19 das 29 prescrições que combinavam anti-hipertensivos, antidepressivos e hipoglicemiantes em pesquisa desenvolvida em Minas Gerais ${ }^{22}$. É notável que, até

Tabela 2. Frequências absoluta e relativa de interações medicamentosas em prescrições da Unidade de Saúde da Família Urbis V, Vitória da Conquista, Bahia, Brasil, julho a dezembro de 2010.

\begin{tabular}{lrrc}
\hline & N & \% & IC $_{95 \%}$ \\
\hline Gravidade da interação & & & \\
Menor ou não significante & 70 & 25,1 & $(20,3-30,4)$ \\
Moderada ou significante & 200 & 71,7 & $(66,2-76,7)$ \\
Maior ou muito significante & 9 & 3,2 & $(1,6-6,8)$ \\
Total & $\mathbf{2 7 9}$ & $\mathbf{1 0 0 , 0}$ & \\
Mecanismo de interação & & & \\
Farmacocinético & 123 & 44,1 & $(38,3-50,0)$ \\
Farmacodinâmico & 110 & 39,4 & $(33,8-45,3)$ \\
Físico-Químico & 0 & 0,0 & $(0,0-1,1)$ \\
Misto/Desconhecido & 39 & 14,0 & $(10,3-18,4)$ \\
Não classificado & 7 & 2,5 & $(1,1-4,9)$ \\
Total & $\mathbf{2 7 9}$ & $\mathbf{1 0 0 , 0 0}$ & \\
\hline
\end{tabular}

o momento, a maioria dos estudos realizados no Brasil sobre interação medicamentosa na atenção primária limitou-se à inclusão de algumas classes terapêuticas e dispuseram de uma pequena população de estudo, dificultando a comparação dos seus resultados com os dados desta pesquisa.

Por outro lado, há mais investigações sobre interações medicamentosas em prescrições da atenção primária em outros países. Lopez-Picazo et al., em estudo realizado na atenção primária da Região da Múrcia (Espanha), encontraram prevalência de interações de 5,8\%, sendo $50,0 \%$ dessas consideradas graves; a interação mais frequente foi entre omeprazol e diazepam ${ }^{17}$. Doubova-Dubova et al., relataram em estudo realizado no México, que cerca de $80 \%$ das prescrições ambulatoriais em pacientes com mais de 50 anos de idade apresentavam pelo menos uma combinação com risco potencial de interação medicamentosa. Nesse estudo, $64,0 \%$ das interações foram classificadas com gravidade moderada e cerca de $40 \%$ das interações medicamentosas envolviam a combinação de anti-inflamatórios não esteroidais com anti-hipertensivos ${ }^{23}$. Fiss et al. avaliaram a ocorrência de interações medicamentosas em prescrições de pacientes inseridos no modelo de atenção multidisciplinar em saúde em áreas rurais da Alemanha. Naquele estudo, foi encontrada frequência de $44,6 \%$ de interações medicamentosas relevantes e as classes mais envolvidas foram os analgésicos, antagonistas dos $\beta$ receptores, inibidores da ECA e alguns minerais ${ }^{24}$. Em dois outros estudos, que propuseram metodologias diferenciadas de determinação de interações, 13,9 a 15,0\% das prescrições com terapia medicamentosa combinada continham interações medicamentosas consideradas de gravidade maior, capazes de desencadear uma reação adversa ao medicamento ${ }^{25,26}$. Divergências entre os resultados encontrados no presente trabalho e os estudos mencionados podem ser atribuídas aos métodos empregados para a classificação de interações e ao perfil da população estudada.

$\mathrm{O}$ mecanismo de interação medicamentosa mais comumente encontrado foi o farmacocinético, representando $45,2 \%$ do total de interações analisadas, seguido do mecanismo farmacodinâmico que representou $40,4 \%$ e, por fim, $14,3 \%$ apresentaram tanto mecanismo de interação misto, unindo interação farmacocinética com farmacodinâmica, quanto desconhecido (Tabela 2). As interações encontradas no trabalho de Coelho e Brum em São Paulo envolveram porcentagem distinta de mecanismo farmacocinéti- 
Tabela 3. Interações medicamentosas mais encontradas e suas consequências potenciais em prescrições da Unidade de Saúde da Família Urbis V, Vitória da Conquista, Bahia, Brasil, julho a dezembro de 2010.

\begin{tabular}{|c|c|c|c|}
\hline Interação medicamentosa & $\%\left(\mathrm{IC}_{95 \%}\right)$ & Efeito adverso potencial & Gravidade \\
\hline Amoxicilina e ibuprofeno & $13,6(10,0-18,0)$ & $\begin{array}{l}\text { Interferência nos níveis } \\
\text { plasmáticos de ambos por } \\
\text { competição em proteína de } \\
\text { ligação e clearence renal }\end{array}$ & $\begin{array}{l}\text { Moderada ou } \\
\text { significante }\end{array}$ \\
\hline Enalapril e hidroclorotiazida & $4,3(2,4-7,2)$ & Hipotensão & $\begin{array}{l}\text { Moderada ou } \\
\text { significante }\end{array}$ \\
\hline Ibuprofeno e omeprazol & $4,3(2,4-7,2)$ & $\begin{array}{l}\text { Aumento do nível ou efeito do } \\
\text { ibuprofeno por interferência do } \\
\text { metabolismo hepático }\end{array}$ & $\begin{array}{l}\text { Menor ou } \\
\text { não } \\
\text { significante }\end{array}$ \\
\hline Sulfametoxazol e trimetoprima & $3,9(2,1-6,8)$ & Arritmia cardíaca & $\begin{array}{l}\text { Moderada ou } \\
\text { significante }\end{array}$ \\
\hline Cefalexina e ibuprofeno & $2,2(0,9-4,4)$ & $\begin{array}{l}\text { Aumento do nível ou efeito do } \\
\text { ibuprofeno por interferência no } \\
\text { clearence renal }\end{array}$ & $\begin{array}{l}\text { Menor ou } \\
\text { não } \\
\text { significante }\end{array}$ \\
\hline Hidroclorotiazida e ibuprofeno & $2,2(0,9-4,4)$ & $\begin{array}{l}\text { Alteração na concentração sérica } \\
\text { de potássio }\end{array}$ & $\begin{array}{l}\text { Moderada ou } \\
\text { significante }\end{array}$ \\
\hline Hidroclorotiazida e propranolol & $2,2(0,9-4,4)$ & $\begin{array}{l}\text { Alteração na concentração sérica } \\
\text { de potássio }\end{array}$ & $\begin{array}{l}\text { Moderada ou } \\
\text { significante }\end{array}$ \\
\hline Ácido acetilsalicílico e hidroclorotiazida & $1,8(0,7-3,9)$ & $\begin{array}{l}\text { Alteração na concentração sérica } \\
\text { de potássio }\end{array}$ & $\begin{array}{l}\text { Moderada ou } \\
\text { significante }\end{array}$ \\
\hline Budesonida e loratadina & $1,8(0,7-3,9)$ & $\begin{array}{l}\text { Aumento do nível ou efeito de } \\
\text { ambos por interferência do } \\
\text { metabolismo hepático e bomba } \\
\text { de efluxo (glicoproteína } \mathrm{P} \text { ) }\end{array}$ & $\begin{array}{l}\text { Moderada ou } \\
\text { significante }\end{array}$ \\
\hline Outras & $63,7(58,0-69,3)$ & Não se aplica & Não se aplica \\
\hline Total & 100,0 & & \\
\hline
\end{tabular}

co e farmacodinâmico deste estudo $(23,4 \%$ e $61,7 \%$, respectivamente), sendo $15,9 \%$ de mecanismo misto. Essa diferença pode ser explicada pelo fato da pesquisa destes autores incluírem apenas antidepressivos, anti-hipertensivos e hipoglicemiantes orais, predominando as interações entre fluoxetina e diuréticos - hidroclorotiazida e furosemida (19,2\%); fluoxetina e inibidores da ECA - captopril e enalapril (10,6\%); e imipramina e $\beta$-bloqueadores - propranolol e atenolol (10,6\%); que apresentam mecanismo de interação farmacodinâmico ${ }^{22}$.

O manejo para prevenção ou resolução das interações medicamentosas potenciais pode ser realizado a partir de parâmetros sugeridos pelas bases de dados consultadas, em que se preconiza: ajuste de dose, quando se recomenda a diminuição ou o aumento da dose dos fármacos envolvidos em interações medicamentosas; monitoramento, quando se determinam as concentrações séricas destes fármacos; precaução, onde o uso concomitante de fármacos envolvidos em interações medicamentosas potenciais é feito com cautela; substituição, quando há uma alternativa terapêutica mais segura e eficaz; resposta terapêutica, quando o monitoramento é realizado a partir da resposta farmacológica de um ou mais fármacos; evitar, quando não é recomendado o uso concomitante dos fármacos; horário, quando se recomenda alterar o horário de administração dos medicamentos; dentre outros ${ }^{27,28}$.

Verificou-se que 8,6\% das prescrições da Unidade de Saúde estudada apresentavam polifarmácia; nesse grupo de prescrições, a frequência de interações foi superior a $80 \%$, valor significativamente maior do que o grupo de prescrições sem polifarmácia $(45,6 \% ; p<0,001)$. Estudo retrospectivo com prescrições ambulatoriais realizado em Taiwan mostrou resultados semelhantes aos deste trabalho, onde $67,7 \%$ dos pacientes com prescrições que apresentavam interação medicamentosa potencial também continham 
quatro ou mais medicamentos prescritos, sugerindo associação entre polifarmácia e interação medicamentosa ${ }^{29}$.

A principal limitação deste estudo refere-se ao fato das prescrições arquivadas na farmácia da Unidade de Saúde não conterem informações sobre idade, sexo, diagnóstico, condições de saúde e coprescrições dos pacientes, impossibilitando, dessa forma, a análise de outras variáveis que predispõem ao risco de ocorrência de interações medicamentosas. A impossibilidade de verificar, seja por análise de prontuário ou acompanhamento dos pacientes, se interações resultaram em reações adversas é outra limitação importante do estudo. As bases de dados utilizadas para detecção de interações medicamentosas (o Drug Interaction Checker - Medscape e o University of Maryland Medical Center Drug Checker Micromedex $\left.{ }^{\circledR}\right)$ não continham informações acerca de alguns fármacos prescritos, como a dipirona, presente em 3,2\% das prescrições analisadas.
Em contrapartida, este é um estudo relevante, já que ajuda a preencher lacuna de conhecimento sobre interação medicamentosa na atenção primária à saúde no país.

\section{Conclusões}

A análise das prescrições oriundas da atenção primária de Vitória da Conquista sugere frequências elevadas de interações medicamentosas potenciais de gravidade moderada e maior. Ademais, a associação encontrada entre polifarmácia e interações reforça a necessidade de acompanhamento farmacoterapêutico mais rigoroso. Cabe ressaltar que o Brasil ainda não possui um sistema informatizado de registros que contemple informações sobre o histórico do paciente em todos os níveis de atenção à saúde, o que dificulta a realização desse tipo de estudo no país.

\section{Colaboradores}

DFL Leão trabalhou na concepção e delineamento, análise e interpretação dos dados e redação do artigo; CS Moura na análise e interpretação dos dados, revisão crítica e aprovação da versão a ser publicada; e DS Medeiros na concepção e delineamento, revisão crítica e aprovação da versão a ser publicada.

\section{Agradecimentos}

Os autores agradecem a Rodrigo Damascena, farmacêutico responsável pela Unidade de Saúde da Família Urbis V e preceptor do PET - Saúde da Família, e aos integrantes da linha de pesquisa "Avaliação de prescrições dispensadas em Unidades de Saúde em Vitória da Conquista (BA)" inserida no PET - Saúde da Família. 


\section{Referências}

1. Hammes JA, Pfuetzenreiter F, Silveira F, Koenig A, Westphal A. Prevalência de potenciais interações medicamentosas droga-droga em unidades de terapia intensiva. Rev Bras Ter Intensiva 2008; 20(4): 349-354.

2. Moura C, Acurcio F, Belo N. Drug-drug interactions associated with length of stay and cost of hospitalization. J Pharm Pharmaceut Sci 2009; 12(3):266-272.

3. Riechelmann RP, Zimmermann C, Chin SN, Wang L, O'Carroll A, Zarinehbaf S, Krzyzanowska MK. Potential drug interactions in cancer patients receiving supportive care exclusively. J Pain Symptom Manage 2008; 35(5):535-543.

4. Kohler GI. Drug-drug interactions in medical patients: effects of in hospital treatment and relation to multiple drug use. Int J Clin Pharmacol Ther 2000; 38(11):504-513.

5. Lima REF, Cassiani SHDB. Interações medicamentosas potenciais em pacientes de unidade de terapia intensiva de um hospital universitário. Rev Latino-Am Enfermagem 2009; 17(2):222-227.

6. Fröhlich SE, Zaccolo AV, da Silva SL, Mengue SS. Association between drug prescribing and quality of life in primary care. Pharm World Sci 2010; 32(6):744-751.

7. Organização Mundial de Saúde. How to investigate drug use in health facilities - selected drug use indicators: Geneva: OMS; 1993.

8. Jankel CA, Fitterman LK. Epidemiology of drugdrug interactions as a cause of hospital admissions. Drug Saf 1993; 9(1):51-59.

9. Cruciol-Souza JM, Thomson JC, Catisti DG. Avaliação de prescrições medicamentosas de um hospital universitário brasileiro. Rev Bras Educ Med 2008; 32(2):188-196.

10. Moura CS, Ribeiro AQ, Starling SM. Avaliação de interações medicamentosas potenciais em prescrições médicas do Hospital das Clínicas da Universidade Federal de Minas Gerais (Brasil). Lat Am J Pharm 2007; 26(4):596-601.

11. Jacobson L, Elwyn G, Robling M, Tudor-Jones R. Error and safety in primary care: no clear boundaries. Family Practice 2003; 20(3):237-241.

12. Horn JR, Hansten PD, Chan LN. Proposal for a new tool to evaluate drug interaction cases. Ann Pharmacother 2007; 41(4):674-680.

13. Medscape. Drug Interaction Checker. [página na Internet]. [acessado 2013 out 27]. Disponível em: http://reference.medscape.com/drug-interactionchecker

14. University of Maryland. Medical Center Drug Checker. Drug Interaction Tool. [página na Internet]. [acessado 2013 out 27]. Disponível em: http:// www.umm.edu/adam/drug_checker.htm

15. Jörgensen T, Johansson S, Kennerfalk A, Wallander M, Svärdsudd K. Prescription drug use, diagnoses, and healthcare utilization among the elderly. Ann Pharmacother 2001; 35(9):1004-1009.
16. Brasil. Ministério da Saúde (MS). Conselho Nacional de Saúde. Resolução nº 196 de 10 de outubro de 1996. Diretrizes e Normas Regulamentadoras de Pesquisas Envolvendo Seres Humanos. Diário Oficial da União 1996; 16 out.

17. López-Picazo JJ, Ruiz JC, Sánchez JF, Ariza A, Aguilera B. Escala de peligro para interacción grave: una herramienta para la priorización de estrategias de mejora em la seguridad de la prescripción en medicina de família. Aten Primaria 2011; 43(5):254-262.

18. Acurcio FA, Perini E, Magalhães SMS, Vieira Terceiro LG, Vieira Filho JM, Coutinho KEOM, Caldeira KV, Cabral LHP, Santos MCF, Abrantes PM, Vale SA, Souza MC. Analysis of medical prescriptions dispensed at health centers in Belo Horizonte, Minas Gerais, Brazil. Cad Saude Publica 2004; 20(1):72-79.

19. Bontempo VL, Coelho MC, Chaves JG, Pereira LAM, Andrade WW, Braccini CM. Análise das prescrições geradas pelas equipes do PSF e das Unidades de Atendimento Imediato, em dois distritos sanitários do município de Contagem/MG. Bol Pneumol Sanit 2003; 11(2):17-24.

20. Asiri YA, Al-Arifi MN. Polypharmacy and patterns in drug prescribing at a primary healthcare centre in the Riyadh region of Saudi Arabia. Int J Pharm Pract 2011; 19(2):123-128.

21. Nicolini P, Nascimento JWL, Greco KV, Menezes FG. Fatores relacionados à prescrição médica de antibióticos em farmácia pública da região Oeste da cidade de São Paulo. Cien Saude Colet 2008. 13(Supl.):689-696.

22. Coelho PV, Brum CA. Interactions between antidepressants and antihypertensive and glucose lowering drugs among patients in the HIPERDIA Program, Coronel Fabriciano, Minas Gerais State, Brazil. Cad Saude Publica 2009; 25(10):2229-2236.

23. Doubova Dubova SV, Reyes-Morales H, TorresArreola LP, Suárez-Ortega M. Potential drug-drug and drug-disease interactions in prescriptions for ambulatory patients over 50 years of age in family medicine clinics in Mexico City. BMC Health Serv Res 2007; 7:147.

24. Fiss T, Ritter CA, Alte D, van der Berg N, Hoffmann W. Detection of drug related problems in an interdisciplinary health care model for rural areas in Germany. Pharm World Sci 2010; 32(5):566-574.

25. Bergk V, Gasse C, Rothenbacher D, Loew M, Brenner $\mathrm{H}$, Haefeli WE. Drug interactions in primary care: impact of a new algorithm on risk determination. Clin Pharmacol Ther 2004; 76(1):85-96.

26. Guthrie B, McCowan C, Davey P, Simpson CR, Dreischulte T, Barnett K. High risk prescribing in primary care patients particularly vulnerable to adverse drug events: cross sectional population database analysis in Scottish general practice. $B M J$ 2011; 342:d3514. 
27. Moura CS. Interações medicamentosas em pacientes hospitalizados: exposição, relação com indicadores de internação e intervenção [tese]. Belo Horizonte: Universidade Federal de Minas Gerais; 2010.

28. Moura C, Prado N, Acurcio F. Potential drug-drug interactions associated with prolonged stays in the intensive care unit: a retrospective cohort study. Clin Drug Investing 2011; 31(5):309-316.

29. Lin CF, Wang CY, Bai CH. Polypharmacy, aging and potential drug-drug interactions in outpatients in Taiwan: a retrospective computerized screening study. Drugs Aging 2011; 28(3):219-225.

Artigo apresentado em 15/11/2012

Aprovado em 17/01/2013

Versão final apresentada em 29/01/2013 